\title{
Téoros
}

Revue de recherche en tourisme

\section{Backpackers et carnets de voyage, récit biographique d'une expérience itinérante}

\section{Stéphane Courant}

Volume 32, numéro 1, 2013

Tourisme des routards

URI : https://id.erudit.org/iderudit/1036660ar

DOI : https://doi.org/10.7202/1036660ar

Aller au sommaire du numéro

Éditeur(s)

Université du Québec à Montréal

ISSN

0712-8657 (imprimé)

1923-2705 (numérique)

Découvrir la revue

Citer cet article

Courant, S. (2013). Backpackers et carnets de voyage, récit biographique d'une expérience itinérante. Téoros, 32(1), 112-121. https://doi.org/10.7202/1036660ar
Résumé de l'article

Travaillant depuis plusieurs années sur les écritures de voyage, je propose dans cet article une analyse transversale des carnets tenus par des backpackers. Objet polymorphe, à la fois écritures ordinaires du voyage et écritures laissant entrevoir une intimité du voyageur, le carnet de voyage nécessite la mise en place d'une méthodologie spécifique - observation participante - pour son obtention ou tout simplement pour sa consultation. Au-delà des caractéristiques significatives de la population témoin et du corpus constitué, j'aborderai succinctement la question de la matérialité de l'objet pour laisser une place significative au contenu du carnet. Ainsi, je soulignerai les thématiques que l'on trouve dans l'ensemble de la production et porterai une attention toute particulière à la question du « je » dans ce corpus. Ainsi, feront jour des interrogations, des affirmations du voyageur que seul le carnet de voyage peut révéler. À travers cette production témoignant d'une expérience quotidienne d'itinérance d'une population difficilement saisissable pour l'anthropologue, ce n'est plus une seule présentation d'un pays qui sera dévoilée, mais un ensemble d'éléments construisant une identité narrative. Le carnet apparaîtra alors comme une trace de voyageur, une trace biographique faisant récit et donnant la pleine mesure de la dimension anthropologique du voyage.
Ce document est protégé par la loi sur le droit d'auteur. L'utilisation des services d’Érudit (y compris la reproduction) est assujettie à sa politique d'utilisation que vous pouvez consulter en ligne.

https://apropos.erudit.org/fr/usagers/politique-dutilisation/ 


\title{
Backpackers et carnets de voyage, récit biographique d'une expérience itinérante
}

\author{
Stéphane COURANT \\ Docteur en anthropologie \\ Membre associé du LISST — Université Toulouse le Mirail \\ couraste@numericable.fr
}

\begin{abstract}
RÉSUMÉ: Travaillant depuis plusieurs années sur les écritures de voyage, je propose dans cet article une analyse transversale des carnets tenus par des backpackers. Objet polymorphe, à la fois écritures ordinaires du voyage et écritures laissant entrevoir une intimité du voyageur, le carnet de voyage nécessite la mise en place d'une méthodologie spécifique — observation participante — pour son obtention ou tout simplement pour sa consultation. Au-delà des caractéristiques significatives de la population témoin et du corpus constitué, j'aborderai succinctement la question de la matérialité de l'objet pour laisser une place significative au contenu du carnet. Ainsi, je soulignerai les thématiques que l'on trouve dans l'ensemble de la production et porterai une attention toute particulière à la question du «je » dans ce corpus. Ainsi, feront jour des interrogations, des affirmations du voyageur que seul le carnet de voyage peut révéler. À travers cette production témoignant d'une expérience quotidienne d'itinérance d'une population difficilement saisissable pour l'anthropologue, ce n'est plus une seule présentation d'un pays qui sera dévoilée, mais un ensemble d'éléments construisant une identité narrative. Le carnet apparaîtra alors comme une trace de voyageur, une trace biographique faisant récit et donnant la pleine mesure de la dimension anthropologique du voyage.
\end{abstract}

Mots-clés : Écriture, culture, identité, initiation, rencontre.

Le carnet de voyage est un objet polymorphe, proche du journal intime mais ayant ses propres particularités. Il fait partie du paquetage de nombreux backpackers qui, chacun à leur manière, essaient de mettre en mots leur expérience quotidienne. Souvent seuls, les voyageurs, se voulant autonomes, livrent au carnet leurs déambulations géographiques et existentielles. Mieux que la photographie, il est une trace d'un voyageur, une trace biographique qui fait récit et donne la pleine mesure de la dimension anthropologique du voyage. En offrant un témoignage singulier par le biais d'une écriture journalière, il apparaît tout à la fois comme une source d'informations unique qui permet de compléter les rares ethnographies portant sur les backpackers (Westerhausen, 2002; Sorensen, 2003). Surtout, il révèle cette capacité d'écriture quotidienne, cette nécessité de se raconter et de créer une identité narrative.

J'expliquerai dans un premier temps la méthodologie employée. Je m'attarderai dans un deuxième temps sur la particularité du corpus tout en soulignant quelques caractéristiques sociologiques de la population étudiée. Afin de mieux saisir l'objet, j'exposerai succinctement quelques particularités du carnet. Suite à cette présentation générale, je dévoilerai l'ensemble des thématiques que l'on trouve systématiquement dans les carnets consultés. En dernier lieu, il sera porté une attention toute particulière aux différentes humeurs ou positionnement du «je» des auteurs. Ainsi, nous verrons qu'à travers les écrits se laissent entrevoir des questionnements, des affirmations et des initiations participant à une construction identitaire. En effet, le carnet de voyage est une retranscription de l'expérience quotidienne du voyageur. Il ne s'agit pas d'un discours à postériori mais d'un récit au jour le jour, laissant apparaître les satisfactions, les interrogations et les certitudes d'un voyageur. Il expose en quelque sorte un témoignage d'une expérience quotidienne in situ. Il ne s'agit pas d'interroger un individu sur sa pratique après coup, mais de lire et de percevoir son expérience de l'intérieur. Jean-Didier Urbain (1991) souligne la difficulté de saisir pour l'anthropologue la population touristique en raison, entres autres, de son statut éphémère mais aussi de la volatilité de son discours - on l'interroge après coup, son discours s'adaptant aux attentes de son interlocuteur. Le carnet de voyage offre un témoignage journalier mais surtout brut et persistant - ce qui est écrit ne sera pas effacé ou modifié. En cela, il s'écarte, par exemple, des blogues de voyage (Courant, 2012a; Enoch, 
2010). En effet, si chacun d'eux livre à sa manière un témoignage du for privé du voyageur, il apparaît essentiel de savoir de quelle nature est ce for privé quand celui-ci est proposé à la vue de tous - les blogues - ou alors qu'il est caché. En cela, il offre un témoignage d'une expérience itinérante de l'intérieur d'une population singulière qui, comme le rappelle l'article de Laurie Murphy (2001), est une population résistante à de nombreuses typologies en raison des disharmonies ou dissonances de leurs pratiques. Ce groupe oblige donc le chercheur à prendre principalement les interactions sociales comme point nodal dans l'élaboration de sa problématique si ce dernier souhaite saisir ses modes de fonctionnement, ses représentations ou plus largement ses us et coutumes.

\section{La méthodologie}

La première difficulté quand on désire étudier un tel matériau est d'établir un corpus suffisamment significatif en nombre et un tant soit peu représentatif. Afin de le constituer, j'ai opté pour une technique de terrain traditionnelle de l'anthropologie : l'observation participante. Éprouvée depuis Malinowski (1985), cette technique consiste à vivre pendant une période significative dans un groupe donné afin de percevoir, de comprendre et d'assimiler les pratiques, les manières de faire et de dire, bref d'adopter un mode de vie précis. Pour ce faire, j'ai, pendant un peu plus de 10 ans, voyagé une à deux fois par an pendant des périodes de 8 à 12 semaines sur les continents asiatique, africain et océanique. Ceci a permis de multiplier les contacts avec un grand nombre de backpackers tenant des carnets de voyage. L'observation participante a facilité ainsi les discussions informelles sur le fait de tenir un carnet, mais surtout a permis d'établir un lien de confiance avec les auteurs afin de pouvoir consulter librement leur ouvrage. En effet, lire un carnet n'est pas chose aussi simple qu'il n'y paraît. Il est, pour la grande majorité des auteurs-voyageurs, synonyme d'écriture privée. Demander à consulter leur production revenait à franchir un seuil, à entrer dans des intimités scripturales qu'ils ne voulaient, à l'origine, ne garder que pour eux.

Afin de compléter l'observation et de tendre vers une herméneutique de l'écriture de voyage, j'ai opté pour la mise en place d'entretiens semi-directifs. C'est ainsi que certains aspects et surtout certains secrets d'écriture ou de rapport à l'écriture ont pu être révélés, comme nous le verrons par la suite dans cet article.

D'un point de vue pratique, je n'aborderai ici que les carnets que j'ai pu consulter de manière prolongée. En effet, quelques backpackers montraient un certain intérêt à mon questionnement, mais ne consentaient à m'ouvrir leur carnet qu'à la seule et unique condition d'être présents à mes côtés. Ainsi, ils ou elles avaient la possibilité de sélectionner les pages et de guider ma lecture. Cette restriction a été le plus souvent formulée par des voyageuses. Se pose alors ici des questions relatives au genre, que ce soit en raison de la différence de sexe entre l'observateur et l'auteur ou que cela soit par le contenu même du texte, leur carnet se rapprochant plus d'un journal intime. Cette question du genre ressurgit de manière éclatante également dans le corpus que j'ai fini par établir. Sur un total de 19 carnets, 4 étaient tenus par des voyageuses. Pour tempérer cette disparité, on peut argumenter que le nombre de femmes voyageant seules est somme toute plus réduit que les hommes. Pour aller plus en avant de cette statistique, il paraît opportun d'interroger la relation entre le genre des auteurs et la nature du texte, un axe qui ne pourra être étudié dans cet article.

Au-delà de cette de cette prédominance des carnets masculin - 15 sur 19 -, il est à noter que tous sont issus d'une même catégorie d'âge - 23-28 ans — et de nationalité française. Tous ont en commun d'être partis ou de partir dans les périodes opportunes telles que vacances scolaires, fin d'études, chômage — inversé. D'autres encore profitent des aides sociales (RMI) pour voyager dans des périodes allant 8 à 15 semaines. Chacun d'eux est parti avec l'objectif de revenir, même si c'est à plus ou moins longue échéance. Il ne s'agit pas d'un départ définitif, c'est un voyage pour un temps donné. Tous font appel ou référence, dans une certaine mesure, au wanderlust (Anderson, 1993), à cet attrait du voyage typique au hobo, à cette aspiration à de nouvelles expériences qui leur permet déjà de sortir de leur quotidien. Ainsi, tous voyagent avec cette envie similaire de prendre du bon temps, un temps pour soi, un temps pour les découvertes mais un temps libre de toute obligation.

Écrire dans un carnet semble également répondre à cette même préoccupation. En effet, tout comme les journaux intimes, les carnets semblent être une écriture ordinaire libre de toute contrainte esthétique et morale. Le choix de tenir un carnet est au premier abord une décision personnelle dictée par des motivations individuelles. La liberté de l'auteur peut apparaître comme un principe commun aux différents backpackers rencontrés sur le terrain. De fait, chacun à leur manière, ces backpackers sont des scribes qui notent les évènements survenus ou du moins certains évènements. Cela interroge sur ce qu'est un évènement en voyage ou, du moins, sur ce qui mérite ou non d'être noté. Comme premier élément de réponse, on peut dire qu'il fixe en quelque sorte le marmonnement du monde ou, comme dirait Georges Pérec (1989 : 12), l'infra-ordinaire du voyage.

En effet, l'intérêt principal de ce genre d'écriture est qu'il livre une quantité non négligeable d'informations. Cependant, en raison de la polymorphie de ce support, il est avant tout nécessaire de donner quelques précisions sur l'objet en lui-même.

Tout d'abord, on peut remarquer que cet objet a une appellation spécifique, celui de carnet de voyage et non de carnet de tourisme. Le carnet est donc celui du voyageur, du nomade moderne, mais jamais celui de la figure négative du touriste. Cela préfigure déjà une distinction entre des pratiques de voyage où l'individuel prime sur le groupe, un élément sur lequel je reviendrai par la suite.

Les maisons d'édition, depuis plusieurs années, ne cessent d'augmenter les parutions de carnets de voyages. Initiées par des auteurs-voyageurs tels que Peter Beard (1996) ou encore comme Titouan Lamazou (1998), ces carnets livrent une transcription, une mise en mots de l'expérience du voyage avec une liberté de ton, d'interprétation et surtout de recherche esthétique. Néanmoins, ces ouvrages n'ont pu voir le jour qu'après une révision complète de l'original, les éloignant de la spontanéité du premier jet. Tous ont subi un travail de réécriture, de sélection et de présentation qui ne correspond plus totalement à une véritable production de voyage. À l'inverse, pour le corpus présenté ici, aucune retouche n'a été effectuée. Cela révèle quelques 
surprises sur les représentations et stéréotypes autours du carnet de voyage. L'une d'elle concerne la qualité du support. Sur les 19 carnets, hormis 2 carnets de moleskine et un carnet de même acabit, tous les autres sont de simples cahiers d'écoliers bon marché ayant un papier au grammage assez faible, une couverture fine, bref un support présentant peu de résistance aux aléas du voyage et à la dureté du climat de nombreuses destinations. Hormis les deux carnets de moleskine qui, par ailleurs, ont été offerts à deux voyageuses, tous les autres backpackers confient qu'ils n'ont pas souhaité investir dans l'achat d'un support plus onéreux et surtout plus résistant, comme me l'a confié un auteur sur son carnet de voyage du Vietnam : «Un cahier, c'est un cahier, j'en ai pris un pas cher, je n'avais pas de budget consacré à ça.» On constate alors qu'il n'y a pas d'équivalence entre l'investissement scriptural qui est produit quotidiennement et l'investissement financier pour l'acquisition d'un support sensé recevoir une écriture quotidienne. Si l'on poursuit sur cette prospective autour de la matérialité du carnet de voyage, on peut noter quelques éléments convergents entre les différents carnets du corpus : ils contiennent une centaine de pages. Sur les 19 carnets, 3 sont totalement remplis d'écritures et d'insertions diverses, le reste des carnets laissant seulement une vingtaine de pages vierges. Ils sont en principe destinés à recevoir les descriptions scripturales et autres ajouts pour une seule destination. Ainsi, le corpus regroupe un ensemble de carnets de voyage allant de différents pays d'Asie, d'Océanie, d'Amérique et d'Afrique. Cependant, un carnet de voyage fait exception, un carnet de moleskine. Ce dernier réunit le récit de deux voyages distincts (Inde et Turquie). Ce carnet, comme le confiera l'auteure, est un ouvrage destiné à regrouper ses différentes expériences itinérantes et qu'elle souhaitait laisser à disposition dans sa bibliothèque. Il est intéressant de noter que la grande majorité des carnets est vouée, au retour de voyage, à disparaître dans les tréfonds d'un placard ou d'une table de chevet. Objet caché, il est une archive que l'on ressort de temps à autres, mais qui ne s'affiche pas au regard de tous. Le lecteur est sélectionné, comme tous me l'ont confié. Les auteurs euxmêmes ont manifesté quelques hésitations à relire leur propre carnet. Il y a, comme le rappelait Goody (1978), dans l'écriture un risque : c'est que l'écriture est une forme semi-permanente et le danger est justement là. C'est que, à la relecture d'un carnet de voyage ou à la vue de ces anciens témoins, les inconséquences, la mauvaise foi, les mensonges peuvent sauter aux yeux. Alors ils sont mis de côté et, parfois, ils sont ressortis à la demande de quelques-uns ou à la suite d'un évènement particulier — d'un déménagement, par exemple.

Cette question du visible et du caché revient également sur un nouvel aspect, celui de l'écriture. En effet, on peut noter que certaines écritures sont difficilement déchiffrables et révèlent non pas un effet de style graphique, mais plutôt une stratégie de camouflage. Ne pas former totalement les lettres ou les condenser les unes contre les autres, homogénéiser les espaces entre mots et lettres, griffonner au lieu de calligraphier convenablement les caractères permet aussi de cacher les fautes d'orthographe, de syntaxe, de rédaction mais aussi les aveux intimes. Le voyageur conçoit de livrer ses secrets mais en les parant ainsi d'une sécurité. Un auteur me l'a révélé lors d'un entretien : «Si mon écriture ressemble à des pâtes [sic] de mouches, c'est aussi pour être sûr que personne ne puisse me lire.» Cette sécurité, il est vrai, ne facilite pas le travail ethnographique, mais illustre aussi toute l'intimité qui peut être notée et avouée le long du voyage.

Deux autres caractéristiques sont à noter concernant le corpus : l'omniprésence de l'écriture et la rareté du dessin. Ainsi cinq carnets sont constitués uniquement d'écriture, aucun croquis, aucune présence d'objets insérés. Nous sommes loin du carnet de voyage ressemblant à un herbier ou du voyageur obligatoirement aquarelliste. Deux carnets ont plus d'une quinzaine de dessins (cartes de la région, plages, couché de soleil, ambiance de rue, visage d'enfant, animaux tels que chat et gecko). Le reste du corpus laisse entrevoir de temps à autres de petites tentatives de dessins griffonnés au stylo, sans couleur, qui sont plus proches d'une illustration schématisée que du dessin appliqué. La grande majorité des backpackers interrogés avouent leur difficulté à dessiner et préferent à cette activité l'insertion d'objets tels que cartes postales, tissus et objets divers. Comme l'a précisé un backpacker: "Je suis nul en dessin, c'est plus facile de coller une carte postale ou d'essayer de décrire un paysage... en plus je n'ai pas de feutre ou de peinture.» Ce genre d'argument est revenu à plusieurs reprises lors des entretiens et demeure assez étonnant quand on sait que le carnet n'est pas destiné à être lu et vu par une autre personne que l'auteur lui-même. On comprend alors l'enjeu de prendre en compte ici toute la dimension de la matérialité du carnet de voyage et de ces nombreuses incidences sur la construction du récit biographique. Cependant, je ne pourrai dans cet article aborder cette dimension que partiellement et transversalement. Il aurait été nécessaire de noter la spécificité du carnet de voyage par rapport, par exemple, au carnet de croquis, au cahier de recettes ou même aux écritures ordinaires, comme l'a fait le Laboratoire d'Anthropologie de Toulouse avec les travaux sous la direction de Daniel Fabre (1993). Toutefois, il apparaît plus opportun de saisir ici quelques spécificités de l'écriture - temps et fréquences - , quelques thèmes clés que l'on retrouve dans l'ensemble des carnets étudiés tels que les rencontres, le rapport à la nature, les impondérables du voyage, mais surtout la question du «je». Ne pas développer pleinement l'aspect matériel du carnet pour privilégier l'aspect textuel ne confine pas obligatoirement au contresens, mais fait appel au sens des priorités face à la richesse d'un matériau très versatile. C'est pourquoi je vais m'attarder maintenant sur les thèmes clés omniprésents dans les carnets.

\section{Les temps d'écriture}

À l'inverse du diariste qui décrit quotidiennement son vécu, le carnettiste - terme désignant les personnes écrivant des carnets de voyage - fait part de son expérience uniquement sur une période déterminée, ayant un début et une fin, celle du temps du voyage. Pour chacun des carnettistes, une discipline quotidienne d'écriture est exigée, mais l'inconfort et les aléas du voyage obligent parfois les auteurs-voyageurs à un report du bilan quotidien. Un autre point commun entre ces deux types d'écrivains du quotidien est le respect de rites scripturaires. Comme le précise Béatrice Didier (1976 : 47), tous les diaristes se plient à une forme d'injonction les obligeant à inscrire pour chaque prise d'écriture la date, l'heure, le lieu (qui au demeurant évolue peu). C'est un peu le rituel d'amorçage de l'écriture. Il 
en est de même pour les carnets de voyage : l'inscription de la date et du lieu est l'élément fondateur pour «ouvrir» l'écriture à la description des événements journaliers. Ils sont les déclencheurs de l'écriture, qu'elle soit simple annotation télégraphique ou récit, ils sont présents comme éléments qui poussent intuitivement à raconter ou du moins à laisser quelques informations sur le déroulement de la journée.

Le rituel scripturaire concerne avant tout une inscription de dates et de lieux. Il participe en quelque sorte à la mise en page du texte, ce qui permet de voir, sans effort de lecture, les différentes périodes du voyage. Certains préférent changer de couleur d'encre pour marquer le changement de jour et de lieu. Dans tous les cas, le carnet est là pour marquer le temps qui s'égrène, qui passe et qui parfois échappe aux auteurs, comme en témoigne ce backpacker qui se retrouve, après plus de trois mois de voyage, à l'aéroport de Bankok pour rentrer chez lui : "Je me suis totalement planté de jour! J'arrive pour enregistrer mon sac et, là, l'hôtesse qui me dit : "Sorry, Saturday is tomorow!" Et là, je lui dis : "Quoi, Saturday is to day." Et elle me dit : "No, today is Thursday?" Putain, je me suis planté dans mon planning, je ne sais pas où! Et me voilà reparti pour passer une journée à attendre mon avion!»

À côté des dates, les noms de villes, de lieux-dits, etc. s'enchaînent les uns après les autres comme les péripéties liées à ces espaces. Les auteurs inscrivent le nom du lieu d'où ils écrivent, comme pour réaliser une première appropriation d'un nom, d'une orthographe. Cette inscription fait partie d'un apprentissage géographique. Chacun s'approprie l'espace traversé en l'inscrivant dans la continuité de son voyage. Écrire le nom de la ville, c'est aussi faire part d'une étape, montrer la progression de l'itinéraire, c'est atteindre le but que l'on s'est fixé. Pour les backpackers, voyager, c'est aussi ça, l'accumulation de lieux, de villes qu'ils se plaisent à lister peu à peu.

\section{Les fréquences d'écritures}

L'inscription des dates permet de relever les fréquences d'écriture, afin de repérer les interruptions de rédaction. Il y a d'une manière générale une régularité quotidienne de l'écriture. Cette constance demande au carnettiste une discipline journalière, mais malgré cela il y a parfois des impossibilités causées par les conditions du voyage. La durée des arrêts peut aller de 1 à 10 jours, voire légèrement plus (à de très rares occasions). Lorsqu'il y a interruption d'écriture, on peut constater que diverses stratégies sont présentes : soit une synthèse ou un résumé est réalisé après coup, tout en précisant les raisons des pauses, soit l'arrêt est implicite, voire caché. Est alors mise en place une stratégie de camouflage. Dans ces cas-là, l'auteur réalise un fauxsemblant d'écriture au présent, car l'obsession principale est de répondre à l'exigence d'une rédaction au quotidien. L'essentiel est qu'à chaque jour corresponde une écriture et que le carnet, tel un agenda, procure son lot d'informations nécessaires à une relecture cohérente. Cette stratégie de camouflage est une forme d'écriture de rattrapage, où le carnettiste en un jour récupère plusieurs jours d'absence de notations. L'ensemble des critères de notation est respecté : inscription de la date et du lieu, changement de couleur du stylo, etc. Il s'agit d'un faux, où le carnettiste fait croire à une continuité. En quelques heures, le même jour, il réalise de mémoire une écriture à postériori. Sans la réalisation d'entretiens semi-directifs avec les carnettistes, rien n'aurait pu révéler cette supercherie. L'auteur a donc rusé, a triché et a consenti sciemment à faire ce petit arrangement pour que persiste un accord tacite qu'il s'est jusque-là évertué à tenir : écrire quotidiennement. Tous les carnettistes ont respecté un pacte avec eux-mêmes, un pacte de carnettistes, c'est-à-dire tenir le carnet jusqu'à la fin du voyage, raconter leur aventure au quotidien même si chacun d'eux s'est arrangé avec la vérité. Le pacte du carnettiste n'inclut pas forcément un pacte de sincérité (Lejeune, 1975). Si dans le corpus étudié tous les carnets sont tenus du début à la fin, la moitié des auteurs ont reconnu qu'ils avaient, lors de voyages antérieurs, essayé de tenir un carnet, mais qu'ils n'avaient pu se tenir à une écriture quotidienne et avaient donc abandonné le projet. L'arrêt a été en quelque sorte explicite. La motivation d'écriture s'est peu à peu émoussée, aucune stratégie de camouflage n'a alors été tentée. La description quotidienne est apparue au fur et à mesure comme un exercice exigeant et contraignant. Le voyage s'est alors poursuivi et le carnet s'est interrompu.

Cette lassitude de la prise de notes est aussi révélatrice d'un paradoxe. En effet, il est troublant qu'au temps du voyage synonyme de temps vacant, de temps non productif économiquement, s'adjoigne une discipline scripturale quotidienne. Tous témoignent que, à un moment donné de leur voyage, ils ont été fatigués ou touchés d'un non-enthousiasme pour l'écriture, d'une exigence ou d'une discipline à maintenir alors qu'ils étaient tous dans un temps libre, un temps oisif. D'ailleurs, on peut remarquer que la densité de notes diminue au bout de 15 à 20 jours dans l'ensemble des carnets. Dans tous les cas, il y a pour tous un infléchissement de la production à un moment donné. On pourrait alors soupçonner qu'une usure ou une fatigue scripturale se fait jour. Plusieurs réflexions peuvent être menées pour comprendre cette évolution, mais il apparaît surtout que les backpackers perçoivent le carnet comme un outil à vocation mémorielle : l'écriture permet de retenir tous les événements survenus. Or, c'est une écriture qui se focalise sur une expérience qui s'érode ou qui perd peu à peu de son attrait avec le temps. L'écriture s'émousse à mesure que les surprises ou les étonnements d'hier deviennent des événements journaliers. Se pose alors la question du renouvellement ou de la motivation pour trouver dans cette expérience qui se répète un nouvel élan pour reprendre quotidiennement la plume.

Ainsi, si l'écriture s'étiole, certains sont tentés de transformer l'exercice de rédaction en un exercice plus récréatif, en transformant un temps le carnet en journal herbier. Le backpacker utilise alors plusieurs techniques, que se soit par le coloriage ou l'insertion de multiples matériaux. Par ce jeu d'accumulation, le backpacker poursuit son œuvre de mise en mémoire du quotidien. De cette façon, on peut trouver un peu de tout collé, inséré : des chèques de voyage, des écorces, des feuilles, des insectes, des tickets, des étiquettes, des tissus, et même des mégots de joint. Ils sont les restes symboliques du quotidien, comme une synthèse des éléments considérés comme emblématiques. Toutes ces petites choses insérées sont de l'ordre du souvenir. Chacun des éléments a une histoire propre, représentant symbolique d'une des étapes du voyage. Tous ces éléments incorporés donnant du volume au carnet, le grossissant, provoquant des bosses et des trous, contribuent à la singularité de l'objet. Le carnet se 
transforme lui aussi avec le voyage, il se gonfle de ces petites pièces prélevées ici et là pour se transformer en un agglomérat de souvenirs, un rébus que seul le carnettiste pourra déchiffrer. Le journal herbier est une technique d'appropriation se libérant des contingences scripturales, donnant libre cours à l'inventivité des auteurs. Il singularise le carnet par les choix effectués allant d'un aspect ludique à la recherche esthétique.

Cependant, il paraît nécessaire de préciser que, si le voyage est souvent l'élément déclencheur d'un volontarisme scriptural pour la plupart des backpackers, tous les voyages ne font pas naître forcément l'envie d'écrire. La spécificité du voyage (être seul ou accompagné), la durée, la destination (toutes les régions n'ont pas un même capital ou une même charge symbolique dans les représentations des voyageurs) apparaissent comme des paramètres significatifs pour faire naître l'envie d'écrire, voire de laisser sortir un génie créatif. Ces types de voyage optimisent les chances qu'un "quelque chose se passe», que des expériences singulières se déroulent, offrant des opportunités d'écriture tant sur le voyage en lui-même que sur le rapport à cette expérience. Au-delà du thème du voyage, c'est l'occasion d'écrire sur soi. Il ne s'agit plus seulement de voyage, mais d'une construction d'un événement ou d'une étape biographique où le voyageur-auteur pourra prendre possession d'un moi qu'il est venu appréhender et tester. C'est une construction du moi qui est proche sur quelques points de celle des diaristes :

Le moi est une sorte d'entité que l'on possède; il appar-

tient au domaine de l'avoir, plus qu'à celui de l'être. D'où

cette étrange dissociation, fréquente chez les diaristes :

l'écrivain veut posséder son moi, qui est comme le plus

profond de lui-même, sa terre fondamentale, mais par

rapport à quoi il a une certaine distance, qui favorise

justement l'écriture, puisqu'il s'agit de "posséder" ce moi

(Didier, 1976 : 137).

Nous sommes alors devant une double représentation liant le carnet comme un outil de construction biographique et le voyage comme un accélérateur d'événements biographiques. Dans tous les cas, le voyage laisse et offre suffisamment de temps et d'expériences pour que le backpacker puisse s'éprouver et se décrire à la fois. Encore faut-il qu'il franchisse les frontières d'une intimité jusqu'alors jamais dépeinte.

\section{Les rencontres}

En dehors de la description de l'environnement proche de l'auteur, il y a une nécessité de marquer, de livrer au carnet les premières interactions provoquées ou subies. Cela peut commencer pour certains par la description des personnes assises à côté d'elles alors qu'ils sont tout juste installés dans l'avion : «Mon voisin est assez volumineux, j'espère qu'il ne va pas s'endormir sur moi après le repas!» Ou bien encore : «J'ai comme voisine une Espagnole qui ne fait que transiter à Abu Dhabi [sic] pour se rendre à Jakarta, dommage car nous venons d'avoir une discussion très riche sur le voyage, elle travaille pour l'ONU, elle parle cinq langues dont parfaitement le français.». D'autres backpackers ne commencent véritablement leur carnet qu'après leur arrivée à destination, notamment en décrivant leurs premières interactions : "J'ai pris le bus pour me rendre dans le centre, j'ai adoré leur façon de récupérer l'argent pour payer le ticket. C'était une jeune Thaïe qui maniait une longue boîte en étain avec une adresse folle, elle fait tinter les pièces en remuant la boîte, donne un ticket qu'elle découpe en la pinçant dans la boîte et tout ça au milieu d'un bus bondé.» Hormis les backpackers se faisant un point d'honneur de ne privilégier et de ne noter que les interactions concernant un Autre, tous les autres backpackers carnettistes décrivent, même de façon succincte, leur première rencontre. Or, si pour beaucoup le voyage commence par un vœu pieu de rencontrer la population locale et la culture hôte, les premières rencontres de fait sont avant tout avec des personnes en transit ou en voyage comme l'auteur. Ainsi, cette carnettiste qui s'empresse de noter juste après son arrivé Kuala Lumpur sa rencontre avec un autre voyageur :

Arrivée à l'aéroport, gros speed, beaucoup trop pour moi! Beaucoup de monde, de circulation, la chaleur, je commençais à paniquer! Heureusement, je suis tombé [sic] sur un autre voyageur, un Allemand assez cool! Nous avons pris le bus local ensemble, ça m’a déstressé [sic] un peu et nous avons convenu de prendre un verre ce soir ensemble dans un bar non loin de nos guesthouses! Le hic, j’ai oublié son prénom.

Ainsi les premiers prénoms - quand ils sont retenus, dans le cas contraire, la nationalité fait office de présentation - ou les premières descriptions de personnes concernent des rencontres relativement brèves de voyageurs ayant comme point commun avec le carnettiste d'être des précaires du lien social, partageant un statut commun, celui d'être voyageur, de faire partie de ces individus ayant laissé leur principal réseau social derrière eux. Loin de la rencontre parfois désirée de cet Autre, la rencontre se cantonne avant tout aux semblables. Cet état de fait se voit en plus accentué quand le backpacker rejoint — volontairement ou non - les réseaux touristiques de backpackers tels que ceux constitués par le truchement de guides comme le Routard ou le Lonely Planet. Ce «Même» (Courant, 2012b; Foucault, 1999) est alors décrit seulement à travers quelques variables aseptisées telles que la nationalité, le parcours réalisé, les voyages antérieurs, etc. Les rencontres sont souvent aussi brèves que l'intimité livrée face à cette rencontre. Il n'y a pas de portrait travaillé avec une volonté de dépeindre l'Autre dans sa diversité, il s'agit plus d'une esquisse que d'un véritable portrait.

Or, ce qui est justement marquant est cette absence d'information. Il y a sous ce silence l'idée égalitariste d'une situation, d'un statut commun qui rappelle la société communautaire ou le communitas de Turner (1990). Les descriptions des voyageurs s'effacent pour ne laisser que des personnes ayant une position sociale similaire : celle du néophyte, du nouvel arrivant dans une région donnée, celle du backpacker se voulant autonome. Il est très rare de connaître le statut d'origine (cadre, instituteur, etc.) des voyageurs mentionnés, comme si le voyage ne produisait pas un contexte justifiant une nécessité d'identité par «les distinctions séculières de rang et de statut» (Turner, 1990 : 97) partagées dans la société d'origine des auteurs. De même, il est très rare de connaître l'âge même approximatif des personnes décrites. Turner $(1990: 98)$ souligne l'importance de ce «grand esprit de camaraderie» présent dans le communitas. Or, les carnets listent de manière exhaustive et quotidienne les occupations : les jeux et les personnes les ayant pratiqués, les discussions (avec comme sujet principal le voyage), les repas, voire les ribotes, le partage des drogues (cannabis, opium), etc., 
bref un ensemble de rites communautaires ou amicaux. Le souci du détail de ces rencontres et de ces instants ludiques partagés est plus précis que le portrait des compagnons de voyage. Ainsi les descriptions des plats (couleur, goût, ingrédients, préparation, contenant, etc.) allant jusqu'au schéma sont beaucoup plus présents qu'un éclairage ou qu'une confidence sur la personne ayant goûté ce plat. L'écriture s'attarde plus sur les détails, les éléments matériels qui participent à la rencontre que sur les personnes rencontrées.

Pourtant, malgré les absences dans la description et la préférence pour certains éléments matériels, la rencontre s'opère. Elle est notée. La question de la communitas appliquée au tourisme est complexe en raison de la variabilité de la population. En fait, le carnet révèle la contradiction entre la représentation classique du voyage comme rencontre obligatoire de l'Autre, et une réalité graphique du voyage qui dépeint surtout le voyage comme une rencontre du Même.

Ce Même est en fait un individu ayant une pratique de cheminement similaire, allochtone comme l'auteur, mais souvent de nationalité différente. Cette similitude de statut n'efface pas pour autant le désir de certains de se démarquer. Or, ce Même qui est présenté dans les carnets, succinctement décrit, avec qui le carnettiste a passé du temps — un temps nécessaire pour déjà justifier cette présence dans le carnet — n'est que très rarement étiqueté comme touriste. En effet, le backpacker ne partage pas les mêmes valeurs que les touristes. Ils ont le voyage grossier, car à leur grand désavantage les touristes laissent échapper une dimension humaine essentielle, celle de l'empathie avec cet Autre, cette population locale qu'ils visitent. À l'inverse, le backpacker a toujours ce désir, plus ou moins révélé, d'une recherche d'altérité, comme l'a montré l'étude de Westerhausen (2002). Il se fait alors juge, il condamne, il incrimine ces comportements d'Occidentaux, d'homo touristicus (Vander Gucht, 2006) qui phagocyte cet Autre tant idéalisé en une simple consommation récréative.

Beaucoup veulent les éviter, mais les routes sont parfois les mêmes et les backpackers ne peuvent échapper à cette confrontation. On perçoit ainsi tout le long des carnets une recherche de singularité, une préoccupation de ne pas faire pareil que les touristes. Ainsi, ce backpacker avoue son compromis entre son idéal et la réalité du voyage en plein Laos :

Direction Vang Vien, c'est un nid à touristes! Ça va encore être TV, guesthouse, des mecs qui fument de partout, qui consomment comme des fous! Je pensais que c'était un bon endroit pour donner un rendez-vous à Marc, mais je me suis planté! C’est bourré de touristes, j'arrive, je choppe Marc, on prend un bus et on se casse direction Luang Prabang.

En effet, cette rencontre peut aussi être un jeu de miroir qui renvoie une similarité de position sociale et de statut partagé : celui d'être un voyageur-touriste. De nombreux backpackers affichent une volonté de se couper de toute communication avec un tel semblable. Ainsi cet autre carnettiste avoue sa fuite devant un autre touriste-voyageur : «J'étais en train de regarder un petit concert de percu [sic] sur la place, concert assez sympa, et voilà que je ne te vois pas un gars avec un Routard [Guide du Routard]! Je croyais être seul, le seul toubab [nom donné aux Occidentaux] ici. Je suis parti dégoûté. Qu'est-ce qu’il fait là?»
La confrontation avec un touriste met à mal un positionnement de distinction et de valorisation de soi quand le voyageur a justement décidé de ne rencontrer que les seuls habitants des régions visitées. Le carnet reproduit cette représentation sociale binaire entre espace touristique dénigré et espace autochtone valorisé.

Tous développent des stratégies d'évitement du touriste, mais tous varient dans l'identification dudit touriste. Il y a de fait un paradoxe entre attirance et répulsion du semblable. En fait, la lecture des carnets permet de saisir que les backpackers carnettistes jouent avec leur statut, les différentes modalités offertes par les structures touristiques, et ne s'enferrent pas tous dans un dénigrement systématique. On comprend alors que le voyage est aussi un ensemble de pratiques culturelles complexes pouvant aller des plus légitimes aux moins légitimes. Ainsi, se mélanger à d'autres touristes est certes une confrontation, mais surtout une dissonance (Lahire, 2004) dans une pratique que le voyageur souhaite idéale, authentique, valorisant les représentations sociales du voyage et non du tourisme. Alors beaucoup se justifient à travers des phrases telles que «cela coûtait moins cher», "c'était plus pratique», «je l'ai fait cette fois-ci, mais c'est la dernière fois », etc. Cet ensemble de fausses notes peuvent arriver dans un voyage, mais, par le ton employé, l'ironie du propos, sont autant de preuves d'une réhabilitation du choix réalisé qu'une distinction à honorer.

Le carnet ne transpose pas uniquement les confrontations avec les touristes. Nous avons en parallèle les remarques et commentaires sur les difficultés interactionnelles avec l'autochtone. Comme en témoigne le rapport difficile de ce voyageur au Vietnam avec les marchands ambulants et leurs nombreuses sollicitations : "Je n'y crois pas, je suis en train d'écrire tranquille, dans un bar, au fond de la salle et voilà que, même ici, ils viennent me vendre un briquet, puis un journal qui date d'il y a une semaine, un autre un hamac ... Peut-on rester tranquille deux minutes... le prochain, je me le fais, je vais le jeter...»

Certes, le rapport à l'Autre et l'intensité interactionnelle varient énormément suivant la destination : entre la NouvelleZélande et le Vietnam, il existe des différences notables. En effet, entre, par exemple, la densité de la sollicitation quotidienne et le positionnement du voyageur, les interactions, les confrontations et les rencontres, etc., tous ces éléments vont demander des disponibilités, des attentes, des motivations, des envies d'immersion ou non, différentes d'une destination à l'autre.

Il existe donc un ensemble de variables dans le rendu de la confrontation entre voyageurs et autochtones. L'intensité des interactions ou la fatigue interactionnelle sont tout autant tributaires des dimensions politiques, économiques, sociales, culturelles, de l'ouverture plus ou moins effective au tourisme que du carnettiste et de sa capacité à mener cette rencontre.

Cependant, on remarque la difficulté, de manière générale, de tous les auteurs à décrire les rencontres avec cet Autre. La description s'avère être un exercice de style peu commun. La présentation est alors assez courte et proche d'une écriture du ressenti, voire de l'impulsif : «Les Laos sont des gens formidables.» De fait, cet Autre reste dans le discours et rarement dans le récit. Il a surtout droit aux poncifs qui noircissent et occupent quelques lignes du carnet. 


\section{Les thèmes abordés}

Les propos se rattachant à la nature et à l'environnement sont omniprésents dans les carnets. Ainsi, le rapport au climat est, pour beaucoup, abordé dès le début de la prise de notes : "Arrivée à Bangkok, une gangue de chaleur et d'humidité, nous [sic] cerne.» Il est un sujet récurrent sur lequel les carnettistes relèvent toujours un élément plus ou moins informatif. Il est en quelque sorte très proche de ces conversations informelles - avec un commerçant du quartier, par exemple le boulanger - où est abordée la question du temps afin de combler une crainte de silence ou de blanc trop pesant. Il est le sujet qui n'apporte pas grand-chose à un quelconque débat, mais qui permet surtout de nourrir l'interaction entre deux individus. Sujet consensuel, il a pourtant une vertu essentielle, celle d'occuper et de donner une contenance aux protagonistes. Que ce soit à l'écrit ou à l'oral, il permet de lancer la conversation ou la description. Il comble un vide. Avec les carnets de voyage, ce rituel d'occupation est semblable : si rien ne se passe vraiment et que le climat ne convient pas, alors quelques mots sont lâchés sur ce sujet banal et si coutumier. Cependant, dans le cas de figure où le climat est la cause de difficultés de réalisation, voire d'annulation d'un projet, alors cet élément naturel peut déclencher des tirades colériques.

Pluie, chaleur, froid, humidité, vent, etc., bref tout le vocabulaire de la météorologie est présent et pèse sur la réalité du voyage. Ainsi, le rapport au corps est en lien permanent avec le climat. L'acclimatation est donc un des autres sujets en rapport avec l'environnement : l'adaptation à une température, à un rythme lié à cette dernière. Le tout, dans une certaine urgence, en raison d'une volonté de respecter un itinéraire souvent établi à l'avance.

Si le temps est un point central des notations, la végétation et les beautés naturelles sont aussi un élément important. Tout comme le climat, elles sont évaluées, dépassant les attentes des carnettistes ou, parfois, incarnant une déception. Elles sont les marques exotiques et identitaires de la région visitée. Paradoxalement, elles sont rarement décrites et laissent surtout à la photographie le privilège de les capter. Elles s'inscrivent plus dans une iconographie que dans un récit, d'où une présence de collage de cartes, de photos ou de dessins illustrant les beautés environnementales naturelles ou résultat d'une activité humaine.

Une autre thématique proche en quelque sorte de la nature et de l'environnement est la nourriture. Elle est le sujet commun à tous les carnets. La nourriture est un point central, répétitif, détaillé et surtout s'attachant à la nouveauté. La profusion des commentaires peut être synthétisée dans ce que Fischler (1990) nomme le système classificatoire entre le consommable et le non consommable. Les backpackers délivrent leurs goûts, leurs appréciations, l'hygiène autour du plat consommé, bref toute une rationalité, un étalonnage de valeurs nutritionnelles, économiques, esthétiques mais ayant une vertu essentielle : celle d'être exotique.

Cette dernière qualité, au-delà du symbolisme, renforce la position du voyageur balancé entre le statut de néophile, prêt à tout tester, à goûter de nouvelles nourritures, et son opposé, le néophobe, montrant une crainte ou une peur de la nouveauté. L'opposition néophobe/néophile est complémentaire de la distinction touriste/voyageur : le voyageur étant celui qui est ouvert à tout et inversement pour le second. D'où une certaine valorisation dans le discours du fait de tester et de s'inscrire sur des chemins gustatifs propres à la population locale. Alors que le touriste s'inscrit plus dans un continuum alimentaire, le désir opposé du voyageur est la surprise, la confrontation. Le thème de l'alimentation reprend cette ambition ou cette stratégie de distinction entre les deux statuts.

Il est à noter la présence de quelques recettes recopiées le long des carnets : cuisson, listes des ingrédients avec parfois des interrogations sur la nature de quelques éléments. La fonction mémorielle d'une recette de cuisine est évidente, elle est un savoir expérimenté et surtout apprécié que le carnettiste transporte jusqu'à chez lui dans le but avoué de retrouver cette expérience gustative une fois revenu. À l'inverse du simple achat de souvenir, elle est un chemin gustatif à partager. Faire un plat, reproduire une recette typique que l'on a pu prendre sur le vif, jouer avec ce savoir transmis de l'autochtone au voyageur valorisent autant le voyage que la personne pouvant réaliser cette préparation à son retour. De fait, cette recette de cuisine a une dimension sociale particulière, elle n'est pas issue d'un ouvrage mais d'une expérience de voyage. Elle est sociale, car elle est partagée, commentée et transmise.

Un autre thème plus succinct, entre nature et culture, est celui consacré au monde animal. Il n'y a pas une unanimité sur ce sujet : on passe de carnets où l'on constate une absence totale de remarques à des carnets aux registres plus riches.

De fait, le carnet de voyage retranscrit à des degrés divers les constructions culturelles inhérentes aux auteurs-voyageurs, mais également les normes et les valeurs que l'auteur-voyageur peut percevoir du pays hôte. Dans ses indignations face aux conditions de vie d'un animal ou devant telle ou telle alimentation, le carnettiste n'exprime que son rapport à la norme, au tabou et à ses propres constructions culturelles.

\section{Les aléas, adaptation et valorisation de soi}

La notion des aléas du voyage comprend toutes les remarques qui sont liées à des impondérables. En effet, les événements imprévus sont toujours notés. Large thématique, les aléas donnent en quelque sorte du piquant à la journée, mais aussi de l'encre au récit quotidien. L'aléa nourrit l'écriture et le récit. Il singularise le voyage et le voyageur, élément perturbateur qui, s'il est réglé, donne une valeur différente, une spécificité à la journée, voire au voyage.

Les aléas sont souvent causés par une circonstance imputée à l'infrastructure de la société : état des routes, transports publics, équipements hôteliers, etc. À l'exception des backpackers visant un aspect pragmatique - comme communiquer ces informations à un guide - les conditions de logement, de transport sont notées laconiquement et même pour certains passées sous silence. Toutefois, lorsqu'un imprévu survient, il est alors le sujet que tous n'omettront pas de signaler. Ainsi, un exemple allie conditions de logement, hygiène, pénurie d'eau : «On est toujours aussi sale et poussiéreux, et bien sûr il n'y a pas d'eau et pas d'électricité [...] hier soir, on s'est lavé avec l'eau des chiottes, ça a l'air crade, mais quand on pue comme des veaux, de l'eau, c'est de l'eau.» L'écriture témoigne, l'auteur souhaite retenir cet événement comme une confrontation à une réalité du voyage. 
Le manque d'eau est en fait une épreuve. C'est une épreuve non souhaitée mais dans laquelle l'auteur-voyageur trouve une opportunité pour tester sa capacité à s'adapter. Il franchit cet événement non sans une certaine satisfaction que l'on devine à la lecture de son témoignage. Alors, on peut se demander si l'adaptation à de telles circonstances n'est pas l'un des véritables enjeux du voyage : être capable de se dépasser afin d'aller au-delà des simples contingences matérielles. Nous sommes alors dans le registre de l'initiation individuelle où les hasards et les accidents du voyage sont autant de défis pour une mise à l'épreuve de soi.

Cependant, tous les aléas ne sont pas systématiquement des épreuves, et tous les carnettistes ne les perçoivent pas comme une expérience pouvant être valorisée, certains vont l'être, d'autres non. Il est probable que chaque aléa pouvant valoriser une capacité d'adaptation est mis en valeur. Dans tous les cas, par leur nature imprévisible, les aléas sont un point important de l'écriture quotidienne : c'est l'aspect exceptionnel, étonnant, qui justifie l'écriture ou qui donne matière à l'écriture. Cela permet de rompre avec le discours quotidien et souvent monotone du voyage. Peut-être que, tout simplement, l'aléa est cette petite chose qui survient, la surprise que le backpacker souhaite secrètement. Après tout, aucune organisation ou structure de tourisme ne peut la mettre en place et on peut alors se demander si ce n'est pas cette crise impromptue qui conduit de fait à la véritable singularité du voyage.

L'aléa est alors l'événement idéal pour une mise en valeur de soi. On assiste à un recyclage - qui n'est rien d'autre qu'une capitalisation - de l'événement impromptu en un élément valorisant le «je» et nourrissant la distinction avec les autres voyageurs.

\section{Les différents «je» du backpacker carnettiste}

Comme nous allons le voir, il y a une inconstance dans l'écriture des backpackers, dans le sens qu'on y trouve selon les jours, une écriture dépourvue de toute implication du «je» et des écritures plus introspectives faisant part des interrogations plus ou moins intimes, des constats opposés tant sur le plan physique que psychologique. Ce sont des écritures livrant des intériorités fluctuantes. C'est un «je» qui évolue, qui passe de l'enthousiasme d'un jour à un lendemain qui déchante. Le carnet livre ainsi des humeurs contingentes qui vont et viennent entre perte, quête, recherche de soi et un sentiment particulier, le sentiment d'existence.

Cette notion de perte est vague, mais largement compréhensible pour toute personne qui a un jour quitté son chez-soi pour partir plus ou moins longtemps dans de nouvelles contrées. Le sentiment qui en ressort est souvent teinté de peur, d'appréhension, de stress et de questionnement sur un quotidien structurant qui s'absente (certes, le sentiment évolue évidemment suivant l'expérience ou le type de voyage). Une fois l'enthousiasme du départ passé, on retrouve très rapidement dans les carnets les premières écoutes de soi, les premières écritures sur soi.

S'il est un domaine où l'on perçoit ce sentiment de perte, c'est sans aucun doute dans la thématique du rapport au corps ou de la «conscience du corps». L'écoute du corps est un point commun avec les diaristes, sauf qu'en voyage l'écoute devient une surveillance accrue.
L'inquiétude de l'auteur se partage entre la conservation de la santé et l'adaptation. La première perte concerne donc cette insouciance du corps qui jusqu'à présent chez soi n'était questionnée que lorsqu'il y avait un problème. Là, tout est surveillé, le corps est mis en écoute, consciencieusement, les backpackers détaillent leurs maux ou leurs risques potentiels de développer une quelconque contamination.

Cette expression de la perte se fait sentir aussi quand un aléa, un incident de parcours, survient. C'est la perte de la sérénité, la perte du calme et de l'équilibre que le voyageur pensait avoir atteints, comme pour ce backpacker qui se rend compte du vol de son vélo:

Je viens de passer une journée très compliquée, ce matin je ne trouve pas le vélo que j'avais attaché juste en bas du bungalow. Alors qu'hier, j'ai passé une super journée à me balader [...] j'ai passé toute la matinée à me prendre la tête avec le loueur. Il voulait que je rembourse, 250 euros! Je dois y retourner demain, il a gardé mon passeport, le patron de la guest m'a conseillé d'aller voir la police touristique, je ne sais pas comment tout ça va finir.

De temps à autre, le sentiment de perte peut se transformer en sentiment d'échec. C'est le résultat de problèmes relationnels, de lien social ne se faisant pas ou ne menant à rien. Toutefois, l'évaluation est là, le backpacker note, il se note, il estime ses capacités, son adaptation : "Je suis fatigué de mon niveau d'anglais, je viens de refuser une invitation pour aller boire un coup... ils parlent trop vite, mon anglais est lamentable, je vais encore ne rien comprendre.» Parfois, certains se perdent dans leur propre estimation de ce qu'ils étaient avant de partir et de celui qu'ils souhaiteraient être à leur retour. Ils se testent et, dans bien des cas, ils ont quand même conquis quelque chose.

Cette notion de conquête est encore une fois une appellation générale qui peut être appréhendée en observant quatre points. Ces derniers permettent de saisir que les carnets de voyage sont des témoignages positifs, comme les journaux intimes, d'une conquête de soi.

Le premier point est similaire à une caractéristique soulignée par Girard, «la décision de tenir un journal» (1963 : 167). Le carnet est donc plus de l'ordre de la volonté que de la pulsion. Cette pratique réclame discipline et assiduité d'écriture, chose nouvelle pour beaucoup, puisque la plupart des carnettistes n'écrivent que dans cette période et rarement en dehors du voyage.

Le deuxième point se révèle quand l'auteur manifeste une satisfaction d'avoir rencontré de nouvelles personnes. Pour beaucoup, le voyage est un moment de rencontre. Pour nos backpackers, rencontrer et partager du temps avec un autre est un enjeu essentiel du voyage, celui de trouver une altérité, celui d'amener les autres à soi. La conquête de soi peut être appréhendée comme la satisfaction d'avoir été capable de faire du lien social pendant une expérience de voyage en solitaire.

Le troisième point peut être illustré par le rapport au corps. À l'inverse du sentiment de perte de soi, on trouve maintenant une affirmation corporelle. C'est le temps des rites de reconstitution et d'une pleine recherche hédoniste. Il y a de nombreux passages sur les satisfactions d'un bien-être ou d'un corps retrouvé ou tout simplement en forme. Le voyage est aussi une pratique de loisir qui engendre de nombreux rites de 
reconstitution. Le thème du bronzage est un thème transversal aux carnets. Il y a toujours quelques remarques sur l'état de la coloration de peau, la satisfaction de prendre des bains de soleil et d'affirmer peu à peu un corps qui change. Le bronzage est un propos récurrent perçu comme un capital à élaborer petit à petit. Comme le souligne l'étude d'Annette Pritchard (2007), il est un souvenir corporel montré, affiché et entretenu au retour comme pendant le voyage. Marqueur principal, pour les zones chaudes, de la durée du séjour, il se mue en marque de distinction.

Au-delà, de cette affirmation corporelle, c'est le thème du rythme qui fait jour. Le voyage est une opportunité de transformer le cadre quotidien de chacun des voyageurs en les influençant tant dans leur conduite que dans leur engagement. Ce nouveau cadre, comme le précise Goffman (1991), entre en jeu alors dans la perception et les représentations de l'individu sur ses pratiques, sur ses envies et sur son devenir. L'auteur peut se laisser convaincre qu'il peut adopter de nouveaux comportements.

L'écriture rend compte de cette conquête de soi qui se concrétise dans ce nouvel équilibre ou cette discipline de vie que le voyage semble avoir insufflé dans la gestion quotidienne des activités. Cette discipline, dans bien des cas, était recherchée et, maintenant, est acquise et surtout acceptée. Elle a permis à beaucoup de ralentir un tant soit peu, l'art de la procrastination.

La conquête de soi, c'est en quelque sorte la satisfaction d'un bien-être conquis, d'un équilibre trouvé, d'une attente avec soi. Faut-il encore que cela dure au-delà de l'enthousiasme du voyage.

\section{La recherche de soi}

Si le journal intime se centralise sur cette question permanente du «soi» ou du «je», il est aisé de comprendre que l'écriture de voyage suggère également une expression de «soi». Contrairement au journal intime, le carnet ne propose pas un seul et unique «je», il y en a plutôt deux sortes : le premier concerne surtout le «soi en voyage» ou le «je voyageur» et le second, le «soi de retour» ou le «je sédentaire».

Si l'on distingue deux groupes, c'est que, bien au-delà de l'idée d'une continuité du soi, le carnettiste trahit une interrogation, un bilan, des prises de décisions qui ne concernent pas seulement une temporalité, mais deux : celle du présent, c'est-àdire du moment où il voyage et écrit, et celle du futur, du retour.

Ainsi, on trouve dans de nombreux carnets en fin de voyage un bilan, un compte rendu. Il s'agit d'écriture comptable où l'on évalue les plus et les moins, en retenant surtout les plus.

Si le ton pour chacun des auteurs reste le même par rapport au reste du carnet, le registre change avec une écriture persuasive argumentée à coup de bilan comparatif : ici-maintenant/chez moi-au retour, dynamisme/apathie, «je voyageur»/«je sédentaire», procrastination/anticipation, etc. L'écriture formule les appétences dans toutes leurs diversités, variant d'un voyageur à un autre

Chacun à leur manière, les carnettistes instaurent un dialogue dans un entre-soi, comme pour espérer que le réalisé ici soit réalisable là-bas. L'écriture a comme fonction de laisser trace d'une volonté dialogique (Morin, 1991), où les décisions d'aujourd'hui peuvent investir les comportements de demain.
Les comptes rendus ainsi dressés profitent d'un enthousiasme ou d'un dynamisme de fin de voyage comme pour faire durer le «je voyageur» en un «je permanent». Il y a ainsi cette dimension d'évolution, de changement, qui est valorisée et que nous retrouvons dans de nombreux ouvrages ou récits de voyage. Dans son ouvrage, Gérard Cogez (2004) dépeint les écrivains voyageurs au $\mathrm{XX}^{\mathrm{e}}$ siècle et souligne que tous ces auteurs savaient qu'après leur voyage ils allaient rentrer : «... mais ils voulaient au moins que ce fût un individu d'une trempe différente qui rentrât. Car il est manifeste que beaucoup d'entre eux n'étaient pas en bons termes avec eux-mêmes au moment de prendre la route» (Cogez, $2004: 30)$.

Au-delà de cette recherche de soi, il y a cette idée sousjacente que le voyage demeure une expérience initiatique, une expérience permettant de changer de statut, voire d'acquérir un nouveau savoir. Faut-il encore savoir lequel. De fait, la question principale, me semble-t-il, est ce rapport à l'initiation. Il y a surtout un souhait, celui de changer, de rentrer chez soi dans une nouvelle peau, dans un rapport à soi différent, plus équilibré. Le voyage est perçu comme un rituel de passage qui, obligatoirement, amène des modifications : une épreuve salvatrice pour certains ou générateur d'un nouveau regard sur soi pour d'autres. Le voyage semble être une expérience que l'on peut investir de ses multiples désirs, envies et mensonges, comme le précise Jean-Didier Urbain dans Secrets de voyage : "Le voyage est une intrigue ou, plus exactement, une mise en intrigue du Monde » (Urbain, 1993 : 31). Je dirais plus qu'il s'agit d'une mise en intrigue de son propre monde, de sa recherche de soi. Au-delà de la question de savoir si, oui ou non, il y a une initiation, il me paraît intéressant de souligner cette fonction attribuée au voyage comme une expérience obligatoirement initiatique. Nombreux sont les carnettistes à le vouloir, à le souhaiter.

\section{Le sentiment d'existence}

Il y a dans ce bilan une dimension symbolique qui va bien au-delà du rituel de reconstitution. Les carnets témoignent aussi des instants de joie profonde. Beaucoup de carnettistes vont faire l'effort d'analyser la raison de cette joie. Ils essayent de la traduire en mots, de comprendre ce qui a déclenché ce sentiment : un paysage, une rencontre, un moment singulier, une expérience donnée qui est alors qualifiée «de magique», «d'unique», «d'extraordinaire», "d'intemporel», etc. Cela fait étrangement écho au livre de Jack Kerouac, Satori à Paris (1993). Dans cet ouvrage, Kerouac explique que, de retour chez lui après un voyage en France, il a subi une sorte d'illumination, un réveil brusque, un satori. Ne sachant pas à quel épisode précis attribuer cette révélation, il va essayer de retranscrire les différents épisodes qui auraient provoqué ce sentiment. L'absence de notes pendant son voyage, l'entremêlement des souvenirs vont l'obliger à refaire son voyage pour essayer de découvrir quels évènements, quelles rencontres ont pu participer à ce sentiment qu'il ne peut que définir par ce terme si général de satori.

L'avantage du carnettiste sur Kerouac, c'est que lui prend des notes quotidiennes et que nombre de carnettistes vont prendre pleine mesure alors de «cet instant magique» où ils se découvrent une vérité, leur vérité, leur sentiment de l'existence. Il ne s'agit en rien d'un sentiment du «Tout-Autre» (Otto, 2001), mais plus d'une réflexion ou d'une mise en mots de ce que 
Raymond Ledrut (1979:78) nommait le sentiment d'existence: "Comment saisir un "sentiment d'existence"? On ne peut pas l'atteindre directement; on doit le reconstruire ou plutôt le construire, car il n'est ni une donnée ni une essence [...] il est, en effet, ce qui rend possible un code où se trouvent, soit distingués soit confondus, l'homme et la nature, le spirituel et le matériel».

Le voyage semble alors l'expérience qui permet tout à la fois de tester le backpacker tant sur son adaptation et ses certitudes que sur son rapport au monde. C'est une expérience ou un événement singulier qui lui permet de se sentir exister grâce à une liberté — ou du moins un sentiment de liberté — qu'il semble pouvoir éprouver à son gré.

\section{Conclusion}

Le carnet de voyage est donc un réceptacle qui peut aussi bien recueillir des récits qui sont à la fois les résumés quotidiens des expériences du voyage et des récits plus intimistes abordant un questionnement ou des affirmations identitaires. Ma demande de consultation des carnets a eu comme première conséquence que tous les backpackers ont relu leur ouvrage. Le temps ayant fait son œuvre, le carnet n'est plus le simple livre comptable recueillant les informations, il est essentiellement ouvrage de souvenirs : souvenirs de voyage, d'épreuves accomplies, d'expériences plus ou moins bien gérées. Il demeure une photographie, un témoignage d'un individu que certains carnettistes avaient totalement oublié. Pourtant chacun a consciencieusement relu son texte, sélectionnant parfois les passages considérés comme trop personnels et me demandant de ne pas les prendre en compte. Le carnet est un instantané d'une expérience, d'une période. C'est une archive personnelle empreinte de nostalgie qui a matérialisé une expérience faite de confrontations, de rencontres et surtout pendant laquelle le «je» s'est bâti. Cette matérialisation d'expériences accumulées est une marque dans la biographie de chaque voyageur. C'est une période repère dans laquelle certains auteurs aiment se replonger pour échapper à quelques vicissitudes du quotidien et pour profiter d'un extrait de vie considéré pour la très grande majorité comme une expérience biographique réussie.

Il y a dans la construction individuelle cette nécessité de trouver et de produire des repères. La représentation sociale du voyage laisse un espace suffisant pour un investissement individuel et le carnet de voyage devient, après coup, un outil de construction biographique. Il n'est plus alors le simple réceptacle, il est objet, symbole d'une expérience qui, pour beaucoup, est un épisode de construction identitaire.

La charge émotionnelle et le poids de l'expérience retranscrits dans le carnet en font, au même titre que les photos souvenirs, un des premiers acteurs de l'idéalisation des voyages. En effet, c'est pendant la période du voyage que beaucoup se sont mis à écrire et se sont tournés vers eux-mêmes pour une écoute plus attentive de leur rapport à soi et au monde. Tenir un carnet participe alors à un processus de connaissance de soi, permettant de faire émerger un savoir acquis grâce à l'expérience du voyage. Le carnet est alors un outil qui accroît «le champ de l'activité critique» (Goody, $1978: 53$ ), et «l'art du commentaire» (Goody, 1978 : 54). On se rend compte alors que les carnets de voyage ne sont pas seulement composés d'écritures présentant de nouveaux horizons, ils laissent entrevoir des interrogations, des introspections, des questionnements existentiels, des écritures qui sont donc tournées vers soi et qui apparaissent être plus verticales qu'horizontales.

\section{Références}

ANDERSON, Nels (1993) Le Hobo, Sociologie du sans-abri, Paris : Éditions Nathan. 319 p.

BEARD, Peter (1996) Photographies, Paris : Éditions Centre National de la Photographie. $144 \mathrm{p}$.

COGEZ, Gérard (2004) Les écrivains voyageurs au XXe siècle, Paris : Éditions Seuil. 229 p.

COURANT, Stéphane (2012a) Approche anthropologique des écritures de voyage, Paris : L'Harmattan. $314 \mathrm{p}$

COURANT, Stéphane (2012b) «Guide de voyage, réceptions et interactions, la lecture comme quête aux similitudes", Revue Mémoire du livre/Studies in Book Culture, vol. 3, nº 2, <http://id.erudit.org/iderudit/1009346ar>.

DIDIER, Béatrice (1976) Le journal intime, Paris : Éditions aux Presses Universitaires Françaises. 205 p.

ENOCH, Yael (2010) «Blogs of Israeli and Danish backpackers to India», Annals of tourism research, vol. 37, n 2, p. 520-536.

FABRE, Daniel (1993) Les écritures ordinaires, Paris : Éditions POL. 374 p.

FISCHLER, Claude (1990) L'homnivore, le goût, la cuisine, le corps, Paris : Éditions Odile Jacob. 440 p.

FOUCAULT, Michel (1999) Les mots et les choses, Paris : Éditions Gallimard. $370 \mathrm{p}$.

GIRARD, Alain (1963), Le journal intime, Paris : Éditions PUF. 638 p.

GOFFMAN, Erving (1991) Les cadres de l'expérience, Paris : Éditions de Minuit. $576 \mathrm{p}$.

GOODY, Jack (1978) La raison graphique, la domestication de la pensée sauvage, Paris : Éditions de Minuit. 274 p.

KEROUAC, Jack (1993) Satori à Paris, Paris : Éditions Gallimard. 157 p.

LEJEUNE, Philippe (1975) Le pacte biographique, Paris : Éditions Seuil. 357 p.

LAHIRE, Bernard (2004) La culture des individus, dissonances culturelles et distinction de soi, Paris : Éditions La Découverte. 777 p.

LAMAZOU, Titouan (1998) Carnets de voyage, Paris : Éditions Nouveaux Loisirs. 159 p.

LEDRUT, Raymond (1979) La révolution cachée, Paris : Éditions Casterman. $180 \mathrm{p}$.

MALINOWSKI, Bronislaw (1985) Journal d'ethnographe, Paris : Éditions Seuil. $301 \mathrm{p}$.

MORIN, Edgar (1991) La méthode 4, Les idées : leur habitat, leur vie, leur mœurs, leur organisation, Paris : Éditions Seuil. $261 \mathrm{p}$.

MURPHY, Laurie (2001) «Exploring social interactions of backpackers», Annals of tourism research, vol. $28, \mathrm{n}^{\circ}$ 1, p. 50-67.

OTTO, Rudolf (2001) Le sacré, Paris : Éditions Petite Bibliothèque Payot. 237 p. PEREC, George (1989) L'infra-ordinaire, Paris : Éditions Seuil. 121 p.

PRITCHARD, Annette (2007) Tourism and Gender: Embodiment, Sensuality and Experience, Cambridge : CABI. $318 \mathrm{p}$.

SØRENSEN, Anders (2003) «Backpackers Ethnography», Annals of tourism research, vol. 30, $\mathrm{n}^{\circ} 4$, p. 847-867.

TURNER, Victor (1990) Le phénomène rituel, structures et contre-structure, Paris : Éditions PUF. 206 p.

URBAIN, Jean-Didier (1993) L'idiot du voyage, histoires de touristes, Paris : Éditions Payot. 353 p.

VANDER GUCHT, Daniel (2006) Ecce Homo Touristicus, Genève : Éditions Labor. $131 \mathrm{p}$.

WESTERHAUSEN, Klauss (2002) Beyond the beach, an ethnography of modern travellers in Asia, Bangkok : Edition White Lotus Co. 272 p. 